\title{
Assessment of Biochemical and Hematological Parameters in the CRF of Before and After Hemodialysis at Martyr Mohammed El-Najjar Hospital in Gaza Strip, Palestine
}

\author{
Mohamad Alhamss ${ }^{1}$, Ahmad Tabash ${ }^{1}$, Abdrabbou Elregeb ${ }^{1}$, Ayman Abu Mustafa ${ }^{2,}$ ", Asmaa Hejazi ${ }^{1}$, \\ Riyad Shaban ${ }^{1}$, Ramy Sarafandi ${ }^{1}$, Yaser Elarja ${ }^{1}$, Nedal Hasanain ${ }^{1}$, Shawgy Elhessy ${ }^{1}$, Faten Allyan ${ }^{3}$ \\ ${ }^{1}$ El Najar Hospital, Ministry of Health, Gaza Strip, Palestine \\ ${ }^{2}$ Palestine College of Nursing, Ministry of Health, Gaza Strip, Palestine \\ ${ }^{3}$ Department of Biochemistry, College of Medical Sciences, Israa University, Gaza, Palestine
}

Email address:

aymanayman20092009@hotmail.com (A. A. Mustafa)

${ }^{*}$ Corresponding author

To cite this article:

Mohamad Alhamss, Ahmad Tabash, Abdrabbou Elregeb, Ayman Abu Mustafa, Asmaa Hejazi, Riyad Shaban, Ramy Sarafandi, Yaser Elarja, Nedal Hasanain, Shawgy Elhessy, Faten Allyan. Assessment of Biochemical and Hematological Parameters in the CRF of Before and After Hemodialysis at Martyr Mohammed El-Najjar Hospital in Gaza Strip, Palestine. American Journal of Laboratory Medicine.

Vol. 6, No. 1, 2021, pp. 8-16. doi: 10.11648/j.ajlm.20210601.12

Received: November 24, 2020; Accepted: December 29, 2020; Published: March 12, 2021

\begin{abstract}
There are limited data on the assessment biochemical and hematological profile in the CRF (CRF) of before and after hemodialysis in the Gaza Strip. The study aimed to evaluating some biochemical parameters and hematological profile in the CRF of before and after hemodialysis at Martyr Mohammed El-Najjar Hospital in Rafah, Gaza Strip, Palestine. Forty-three (end-stage renal disease) ESRD patients, aged 20-70 years old who were referred to Martyr Mohammed Youssef Al-Najjar Hospital in Rafah for the hemodialysis department. 43 apparently healthy individuals matched for ages were used as a control group for comparisons. The results showed that after-dialysis higher statistically significant in $\mathrm{Hb}$, RBC, HCT, PLT, AST, ALT, ALP and Ca level than before-dialysis. While Ph, WBC, RDW, creatinine (Cr.), Urea was lower statistically significant in after hemodialysis than before-dialysis $(\mathrm{P}<0.05)$. theses indicate to :chronic kidney disease shows abnormal hematological parameters, precisely reduced levels of RBC count, hemoglobin, hematocrit, and platelet count, in addition, before-dialysis patients are more anemic and thrombocytopenic than after-dialysis patients and this is may indicate of lack of blood pressure and type 2 diabetes mellites (T2DM) control in these patients. AST\& ALT activities vary in concentration before and after hemodialysis and make the after-dialysis patients are higher than before-dialysis.
\end{abstract}

Keywords: Biochemical Parameters, Hematological Profile, CRF, Martyr Mohammed El-Najjar Hospital, Rafah, Gaza Strip, Palestine

\section{Introduction}

The kidney is a vital organ that eliminates waste and extra water from the blood and preserves electrolytes balance such as sodium, potassium, and calcium normally in the body [1] The kidneys also control blood pressure and stimulate erythropoiesis [2].

Kidney failure, also known as End-stage renal disease means kidneys are only functioning from $10-15 \%$ of the renal capacity and with GFR of $15 \mathrm{ml} / \mathrm{min}$ or less [3]. It is can occur suddenly or slowly [4].
There are several options for treating kidney failure including kidney transplant, HD (HD) and peritoneal hemodialysis [5]. HD is a process of clearing the blood and eliminating waste (creatinine and urea) from the blood circulation and correct electrolyte imbalance [6].

The hematological disturbance is common in chronic kidney disease (CKD) due to relative deficiency/reduction in erythropoietin (EPO) production and other factors such as increased hemolysis, suppression of bone marrow hormones (erythropoiesis), and hematuria and decreasing loss blood from gastrointestinal [7]. 
Creatinine is waste product produced in the muscles by the changes of creatine and phosphocreatine. $\mathrm{Cr}$ in blood indicated to endogenous production. Therefore it is an good indicator to renal function test. Urea playing a vital role in the metabolism of nitrogen compounds and Urea is a major excretory product of protein metabolism. $90 \%$ of it is excreted through the kidneys and kidney disease is associated with accumulation of urea in the blood. Urea in plasma concentration is determined by renal function test and indicated to the amount of protein catabolism [8].

Calcium is one of the key elements to be considered in patients on hemodialysis due to its relationship with cardiovascular risk [9]. Accurate assessment of calcium balance in patients on HD (HD) is crucial. A negative calcium balance can cause hemodynamic instability, muscle cramps, secondary hyperparathyroidism and loss of BMD. A positive calcium balance may lead to hypercalcemia, vascular calcification and increased cardiovascular morbidity and mortality [10].

An elevated in the risk of cardiovascular disease (CVD) morbidity due to hyperphosphatemia in patients undergoing HD has been demonstrated [11].

CRF is associated with increased levels of some biochemical parameters and decreased others. Hence; we evaluated the before-dialysis and after-dialysis values renal biomarkers in CRF patients undergoing hemodialysis to elucidate the effect of hemodialysis on hemodialysis patients.

\section{Objectives of the Study}

\subsection{The Main Objective of This Study}

The goal of the study is to assess some parameters and hematological profile in the CRF of before and after hemodialysis at Martyr Mohammed El-Najjar Hospital in Rafah, Gaza Strip, Palestine.

\subsection{Subjects and Methods}

Study Design was a case-control study.

Target Population were CRF patients aged (20-70) old years and under hemodialysis among Rafah patients.

\subsection{Study Setting}

The researcher was visiting the department of hemodialysis for easy collection.

\subsection{Sample Size}

The population of the study includes $43 \mathrm{CRF}$ patients, aged 20-70 years old who were referred to Martyr Mohammed Youssef Al-Najjar Hospital in Rafah for the hemodialysis department. 43 apparently healthy individuals matched for ages were used as a control group for comparisons.

\subsection{Distribution of Samples}

The study will conduct in the hemodialysis department in Martyr Mohammed Youssef Al-Najjar Hospital in Rafah.

\subsection{Period of the Study}

The study will carry out during the period from September 2019 to September 2020.

\section{Inclusion and Exclusion criteria}

\subsection{Inclusion Criteria Include the Case}

1. All the patients suffering from chronic kidney disease

2. Aged (20-70) years old.

\subsection{Inclusion Criteria Include of Control}

CRF individuals aged between 20 to 70 years old.

\subsection{Exclusion Criteria}

1. Patients under 20 years of age.

2. Patients more than 70 years of age.

3. Patients with schizophrenia, obesity, cancer, Alzheimer's disease,

4. vascular dementia, and hepatitis.

5. Pregnant and lactating women.

\subsection{Ethical Consideration}

A Consent form was obtaining from the participants after provided with a full explanation of the intended study.

\section{Data Collection}

\subsection{Pilot Study}

A pilot study will conduct for five participants to identify any problem in research design before starting for data collection

\subsection{Questionnaire Interview}

An interview will use for filling in questionnaires that designed for matching the study need of the participants. All interviews will conduct face to face by the researcher. During the survey, the interview will explain any of the questions that were not clear. The questionnaire will base on the questions of previous studies with some modifications. The questionnaire will include questions on the socio-demographic data e.g. age, university level.

\subsection{Sampling and Study Parameters}

Blood was collected in a plain tube and whole blood was collect and test for two hours. The serum is stable 4 days at $2{ }^{\circ} \mathrm{C}$ or 24 hours at room temperature $\left(15-30^{\circ} \mathrm{C}\right)$. The hemolysis of the sample is not applicable. Allow sample tubes to reach room temperature $\left(15^{\circ} \mathrm{C}\right)$ before performing the assay. Also, the complete blood count will collect in the EDTA tube.

All tests will conduct twice; the first time before-dialysis in case. The second time, after-dialysis treatment in the case.

\subsection{Statistical Analysis}

Statistical analyses will form with SPSS version 22. 


\section{Results}

Basline characteristics of the participants

Table 1 shows that $14 \%$ of cases and controls aged between $20-30$ years, $9 \%$ vs. $12 \%$ aged between $31-41$ years, $21 \%$ vs. $23 \%$ aged between $42-52$ years, $33 \%$ vs. $35 \%$ aged between 53-63 years and $23 \%$ vs. $16 \%$ aged between 64-74 years $(\mathrm{P}=0.9)$. In addition, $30 \%$ of cases and $28 \%$ of controls had primary school education, $47 \%$ vs. $44 \%$ had secondary school education and $23 \%$ vs. $28 \%$ had a university education $(\mathrm{P}=0.9)$. Furthermore, $53 \%$ of cases were males, $47 \%$ were females, and $60 \%$ of controls were males and $40 \%$ were females $(\mathrm{P}>0.5)$.

Table 1. Socio-demographic characteristics of the participants.

\begin{tabular}{|c|c|c|c|c|c|c|c|}
\hline \multirow{2}{*}{ Variable } & \multirow{2}{*}{ Category } & \multicolumn{2}{|l|}{ Case } & \multicolumn{2}{|c|}{ Control } & \multirow{2}{*}{$\mathbf{X}^{2}$} & \multirow{2}{*}{ p-value } \\
\hline & & No. & $\%$ & No. & $\%$ & & \\
\hline \multirow{5}{*}{ Age (years) } & $20-30$ & 6 & 14 & 6 & 14 & \multirow{5}{*}{0.7} & \multirow{5}{*}{0.9} \\
\hline & $31-41$ & 4 & 9 & 5 & 12 & & \\
\hline & $42-52$ & 9 & 21 & 10 & 23 & & \\
\hline & $53-63$ & 14 & 33 & 15 & 35 & & \\
\hline & $64-74$ & 10 & 23 & 7 & 16 & & \\
\hline \multirow[t]{2}{*}{ Level of education } & Secondary & 20 & 47 & 19 & 44 & \multirow[t]{2}{*}{0.2} & \multirow[t]{2}{*}{0.9} \\
\hline & University & 10 & 23 & 12 & 28 & & \\
\hline \multirow{2}{*}{ Gender } & Male & 23 & 53 & 26 & 60 & \multirow{2}{*}{0.4} & \multirow{2}{*}{0.5} \\
\hline & Female & 20 & 47 & 17 & 40 & & \\
\hline
\end{tabular}

Table 2 shows that there were significant differences in $\mathrm{Hb}$ level between (cases) before-dialysis (mean $=8.7$ ) and control $($ mean $=13.3)$, P-value 0.001, which means that $\mathrm{Hb}$ level was significantly lower among before-dialysis compared to control. Also, there were significant differences in $\mathrm{RBC}$ between cases $($ mean $=3.1)$ and controls (mean = 5.0), P-value 0.001 , which means that RBC was significantly lower among cases compared to controls. In addition, there were significant differences in WBCs between cases (mean = 4.7) and controls (mean $=5.8)$, P-value 0.001, which means that WBCs were significantly lower among cases compared to controls. The results also show that there were significant differences in HCT level between cases (mean = 27.4) and controls $($ mean $=39.9)$, P-value 0.000 , which means that the HCT level was significantly lower among cases compared to controls. Furthermore, there were significant differences in $\mathrm{MCH}$ level between cases $($ mean $=27.9)$ and controls (mean = 29.1), P-value 0.005, which means that $\mathrm{MCH}$ level was significantly lower among cases compared to controls. Also, there were significant differences in the MCHC level between cases $($ mean $=31.7)$ and controls $($ mean $=32.7)$, Pvalue 0.005 , which means that the MCHC level was significantly lower among cases compared to controls. In addition, there were significant differences in PLT between cases $($ mean $=126.3)$ and controls $($ mean $=264.7)$, P-value 0.001 , which means that PLT was significantly lower among cases compared to controls. On the other hand, there were statistically insignificant differences between cases and controls in levels of MCV $(\mathrm{P}=0.873)$ and $\operatorname{RDW}(\mathrm{P}=1.000)$.

Table 2. Comparison of CBC between before-dialysis and controls.

\begin{tabular}{lllll}
\hline \multirow{2}{*}{ Variables } & before-dialysis $(\mathbf{n}=\mathbf{4 3})$ & Controls $(\mathbf{n}=\mathbf{4 3})$ & Mean differences & t value \\
\cline { 2 - 5 } & Mean \pm SD & Mean \pm SD & -4.6 & -17.005 \\
$\mathrm{Hb}(\mathrm{g} / \mathrm{dl})$ & $8.7 \pm 1.5$ & $13.3 \pm 1.01$ & -1.9 & 0.001 \\
$\mathrm{RBC}\left(10^{9} / \mu \mathrm{L}\right)$ & $3.1 \pm 0.6$ & $5.0 \pm 0.04$ & -1.1 & -20.209 \\
$\mathrm{WBC}\left(10^{9} / \mathrm{L}\right)$ & $4.7 \pm 1.6$ & $5.8 \pm 0.7$ & -12.6 & -4.009 \\
$\mathrm{HCT}(\%)$ & $27.4 \pm 4.8$ & $39.9 \pm 3.0$ & 0.2 & -14.505 \\
$\mathrm{MCV}(\mathrm{fL})$ & $87.6 \pm 7.9$ & $87.4 \pm 0.8$ & -1.2 & 0.160 \\
MCH $(\mathrm{pg})$ & $27.9 \pm 2.7$ & $29.1 \pm 0.8$ & -0.98 & -2.863 \\
MCHC $(\mathrm{g} / \mathrm{dl})$ & $31.7 \pm 0.7$ & $32.7 \pm 0.8$ & 0.001 & -5.822 \\
RDW $(\%)$ & $13.6 \pm 0.9$ & $13.6 \pm 0.5$ & 0.001 & 0.005 \\
PLT $(10 / \mathrm{L})$ & $126.3 \pm 47.8$ & $264.7 \pm 17.7$ & -138.4 & 0.001 \\
\hline
\end{tabular}

Table 3 illustrated that there were significant differences in $\mathrm{Hb}$ level between (cases) after-dialysis (mean $=9.4)$ and control (mean $=13.3)$, P-value 0.001, which means that $\mathrm{Hb}$ level was significantly lower among before-dialysis compared to control. Also, there were significant differences in RBC between cases $($ mean $=3.4)$ and controls (mean = 5.0), P-value 0.001 , which means that RBC was significantly lower among cases compared to controls. In addition, there were significant differences in WBCs between cases (mean $=$ 4.5 ) and controls (mean $=5.9$ ), P-value 0.001, which means that WBCs were significantly lower among cases compared to controls. The results also show that there were significant differences in HCT level between cases $($ mean $=29.7)$ and controls $($ mean $=39.9)$, P-value 0.000 , which means that the HCT level was significantly lower among cases compared to controls. Furthermore, there were significant differences in 
$\mathrm{MCH}$ level between cases $($ mean $=27.9)$ and controls (mean = 29.1), P-value 0.005, which means that $\mathrm{MCH}$ level was significantly lower among cases compared to controls. Also, there were significant differences in $\mathrm{MCHC}$ level between cases $($ mean $=31.8)$ and controls $($ mean $=32.7)$, P-value 0.005 , which means that the MCHC level was significantly lower among cases compared to controls. In addition, there were significant differences in PLT between cases (mean = 119.6) and controls (mean $=264.7$ ), P-value 0.001, which means that PLT was significantly lower among cases compared to controls. On the other hand, there were statistically insignificant differences between cases and controls in levels of $\operatorname{MCV}(\mathrm{P}=0.937)$ and $\operatorname{RDW}(\mathrm{P}=0.417)$.

Table 3. Comparison of $C B C$ between after-dialysis and controls.

\begin{tabular}{|c|c|c|c|c|c|}
\hline \multirow{2}{*}{ Variables } & Post-dialysis $(n=43)$ & Control $(n=43)$ & \multirow{2}{*}{ Mean differences } & \multirow{2}{*}{ t value } & \multirow{2}{*}{ P-value } \\
\hline & Mean \pm SD & Mean \pm SD & & & \\
\hline $\mathrm{Hb}(\mathrm{g} / \mathrm{dl})$ & $9.4 \pm 1.9$ & $13.3 \pm 1.01$ & -3.9 & -12.112 & 0.001 \\
\hline $\operatorname{RBC}\left(10^{9} / \mu \mathrm{L}\right)$ & $3.4 \pm 0.8$ & $5.0 \pm 0.04$ & -1.6 & -14.013 & 0.001 \\
\hline WBC $\left(10^{9} / \mathrm{L}\right)$ & $4.5 \pm 1.7$ & $5.9 \pm 0.7$ & -1.5 & -5.389 & 0.001 \\
\hline $\operatorname{HCT}(\%)$ & $29.7 \pm 5.9$ & $39.9 \pm 3.0$ & -10.3 & -10.168 & 0.000 \\
\hline MCV (fL) & $87.5 \pm 8.1$ & $87.4 \pm 0.8$ & 0.1 & .079 & 0.937 \\
\hline MCHC (g/dl) & $31.8 \pm 0.8$ & $32.7 \pm 0.8$ & -0.91 & -5.098 & 0.001 \\
\hline RDW (\%) & $13.4 \pm 1.2$ & $13.6 \pm 0.5$ & -0.16 & -.816 & 0.417 \\
\hline $\operatorname{PLT}\left(10^{9} / \mathrm{L}\right)$ & $119.6 \pm 40.7$ & $264.7 \pm 17.7$ & -138.4 & -17.820 & 0.001 \\
\hline
\end{tabular}

Table 4 shows that there were significant differences in $\mathrm{Hb}$ level between before-dialysis $($ mean $=8.7)$ and after-dialysis $($ mean $=9.4)$, P-value 0.001, which means that Hb level was significantly lower among before-dialysis compared to afterdialysis. Also, there were significant differences in RBC between before-dialysis (mean $=3.1$ ) and after-dialysis (mean $=3.4)$, P-value 0.001, which means that RBC was significantly lower among before-dialysis compared to afterdialysis. In addition, there were significant differences in WBCs between before-dialysis (mean $=4.7$ ) and afterdialysis (mean $=4.5$ ), P-value 0.001, which means that WBCs were significantly elevated among before-dialysis compared to after-dialysis. The results also show that there were significant differences in the HCT level between before-dialysis $($ mean $=27.4)$ and after-dialysis $($ mean $=$
29.7), P-value 0.000, which means that HCT level was significantly lower among before-dialysis compared to afterdialysis. Furthermore, there were significant differences in the RDW level between before-dialysis $($ mean $=13.6)$ and after-dialysis $($ mean $=13.5)$, P-value 0.031 , which means that RDW level was significantly elevated among before-dialysis compared to after-dialysis. In addition, there were significant differences in PLT between before-dialysis (mean $=126.3$ ) and after-dialysis (mean $=119.6$ ), P-value 0.001, which means that PLT was significantly elevated among beforedialysis compared to after-dialysis. On the other hand, there were statistically insignificant differences between cases and controls in levels of others red blood cells incidence $(\mathrm{P}>0.05)$.

Table 4. Comparison of $C B C$ between before-dialysis and after-dialysis.

\begin{tabular}{|c|c|c|c|c|c|}
\hline \multirow{2}{*}{ Variables } & before-dialysis $(n=43)$ & Post-dialysis $(n=43)$ & \multirow{2}{*}{ Mean differences } & \multirow{2}{*}{$\mathrm{t}$ value } & \multirow{2}{*}{$P$-value } \\
\hline & $\operatorname{Mean} \pm$ SD & Mean \pm SD & & & \\
\hline $\mathrm{Hb}(\mathrm{g} / \mathrm{dl})$ & $8.7 \pm 1.5$ & $9.4 \pm 1.9$ & 0.7 & 5.107 & 0.001 \\
\hline $\mathrm{RBC}\left(10^{9} / \mu \mathrm{L}\right)$ & $3.1 \pm 0.62$ & $3.4 \pm 0.8$ & 0.3 & 5.458 & 0.001 \\
\hline WBC $\left(10^{9} / \mathrm{L}\right)$ & $4.7 \pm 1.7$ & $4.5 \pm 1.6$ & -0.2 & -1.872 & .068 \\
\hline HCT (\%) & $27.4 \pm 4.8$ & $29.7 \pm 5.9$ & 2.3 & 5.188 & 0.000 \\
\hline MCV (fL) & $87.6 \pm 8.1$ & $87.5 \pm 7.9$ & -0.1 & -1.009 & $0 . .319$ \\
\hline $\mathrm{MCHC}(\mathrm{g} / \mathrm{dl})$ & $31.7 \pm 0.7$ & $31.8 \pm 0.8$ & 0.1 & 0.829 & 0.412 \\
\hline RDW (\%) & $13.6 \pm 0.7$ & $13.5 \pm 0.9$ & -0.079 & -2.188 & 0.031 \\
\hline $\operatorname{PLT}\left(10^{9} / \mathrm{L}\right)$ & $126.3 \pm 47.8$ & $119.6 \pm 40.7$ & 3.3 & 2.766 & 0.007 \\
\hline
\end{tabular}

The significance of difference was checked by t-test (compare all case and control), significant at $\mathrm{P} \leq 0.05$.

Table 5 shows that there were significant differences in $\mathrm{Cr}$ level between (cases) before-dialysis (mean $=8.5$ ) and control (mean $=0.8)$, P-value 0.001 , which means that $\mathrm{Cr}$ level was significantly elevated among before-dialysis compared to control. Also, there were significant differences in urea between cases $($ mean $=150.8)$ and controls $($ mean $=$ 35.6), P-value 0.001, which means that urea were significantly elevated among cases compared to controls. In addition, there were significant differences in AST between cases $($ mean $=20.1)$ and controls $($ mean $=28.6)$, P-value 0.001, which means that AST was significantly lower among cases compared to controls. The results also show that there were significant differences in ALT level between cases $($ mean $=20.7)$ and controls $($ mean $=29.1)$, P-value 0.000, 
which means that ALT level was significantly lower among cases compared to controls. Furthermore, there were significant differences in the alkaline phosphatase (ALP) level between cases $($ mean $=450.3)$ and controls (mean $=$ 99.2), P-value 0.005, which means that the ALP level was significantly elevated among cases compared to controls. Also, there were significant differences in Ca level between cases $($ mean $=8.9)$ and controls $($ mean $=9.7)$, P-value 0.005, which means that $\mathrm{Ca}$ level was significantly lower among cases compared to controls. In addition, there were significant differences in phosphorus level between cases $($ mean $=4.9)$ and controls $($ mean $=3.9)$, P-value 0.001, which means that phosphorus was significantly elevated among cases compared to controls.

Table 5. Comparison of some biochemical parameters between before-dialysis and control.

\begin{tabular}{|c|c|c|c|c|c|}
\hline \multirow{2}{*}{ Variables } & before-dialysis $(n=43)$ & Control $(n=43)$ & \multirow{2}{*}{ Mean differences } & \multirow{2}{*}{ t value } & \multirow{2}{*}{$P$-value } \\
\hline & Mean \pm SD & Mean \pm SD & & & \\
\hline Creatinine (mg/dL) & $8.5 \pm 1.9$ & $0.8 \pm 0.1$ & 7.7 & 26.842 & 0.001 \\
\hline Urea (mg/dL) & $150.8 \pm 35.8$ & $35.6 \pm 2.5$ & 115.3 & 21.069 & 0.001 \\
\hline AST (U/L) & $20.1 \pm 9.0$ & $28.6 \pm 1.8$ & -8.6 & -6.122 & 0.001 \\
\hline $\operatorname{ALT}(\mathrm{U} / \mathrm{L})$ & $20.7 \pm 16.3$ & $29.1 \pm 2.1$ & -8.5 & -3.392 & 0.001 \\
\hline $\operatorname{ALP}(\mathrm{U} / \mathrm{L})$ & $450.3 \pm 437.0$ & $99.2 \pm 5.4$ & 351.0 & 5.267 & 0.001 \\
\hline $\mathrm{P}(\mathrm{mg} / \mathrm{dL})$ & $4.9 \pm 0.7$ & $3.9 \pm 0.1$ & 1.0 & 9.668 & 0.001 \\
\hline
\end{tabular}

Table 6 shows that there were significant differences in $\mathrm{Cr}$ level between (cases) after-dialysis (mean $=3.9)$ and control $($ mean $=0.8)$, P-value 0.001, which means that $\mathrm{Cr}$ level was significantly elevated among after-dialysis compared to control. Also, there were significant differences in urea between cases $($ mean $=67.1)$ and controls $($ mean $=35.6)$, Pvalue 0.001 , which means that urea was significantly elevated among cases compared to controls. In addition, there were significant differences in AST between cases (mean $=$ 24.8) and controls (mean $=28.6$ ), P-value 0.019, which means that AST was significantly lower among cases compared to controls. Furthermore, there were significant differences in the ALP level between cases (mean = 484.4) and controls $($ mean $=99.2)$, P-value 0.001, which means that the ALP level was significantly elevated among cases compared to controls. Also, there were significant differences in $\mathrm{Ca}$ level between cases $($ mean $=9.2)$ and controls $($ mean $=$ 9.7), P-value 0.001, which means that $\mathrm{Ca}$ level was significantly lower among cases compared to controls. In addition, there were significant differences in phosphorus level between cases $($ mean $=4.0)$ and controls $($ mean $=3.9)$, P-value 0.004, which means that phosphorus was significantly elevated among cases compared to controls. On the other hand, there were statistically insignificant differences between cases and controls in levels of ALT $(\mathrm{P}=$ $0.161)$

Table 6. Comparison of some biochemical parameters between after-dialysis and control.

\begin{tabular}{|c|c|c|c|c|c|}
\hline \multirow{2}{*}{ Variables } & Post-dialysis $(n=43)$ & Control $(n=43)$ & \multirow{2}{*}{ Mean differences } & \multirow{2}{*}{ t value } & \multirow{2}{*}{$P$-value } \\
\hline & $\operatorname{Mean} \pm$ SD & Mean \pm SD & & & \\
\hline Creatinine (mg/dL) & $3.9 \pm 1.0$ & $0.8 \pm 0.1$ & 3.1 & 21.034 & 0.001 \\
\hline AST (U/L) & $24.8 \pm 10.3$ & $28.6 \pm 1.8$ & -3.8 & -2.395 & 0.019 \\
\hline $\operatorname{ALT}(\mathrm{U} / \mathrm{L})$ & $25.1 \pm 18.6$ & $29.1 \pm 2.1$ & -4.0 & -1.414 & 0.161 \\
\hline $\operatorname{ALP}(\mathrm{U} / \mathrm{L})$ & $484.4 \pm 459.2$ & $99.2 \pm 5.4$ & 385.2 & 5.500 & 0.001 \\
\hline $\mathrm{P}(\mathrm{mg} / \mathrm{dL})$ & $4.0 \pm 0.4$ & $3.9 \pm 0.1$ & 0.2 & 2.923 & 0.004 \\
\hline
\end{tabular}

Table 7 shows that there were significant differences in $\mathrm{Cr}$ level between before-dialysis $($ mean $=8.5)$ and after-dialysis $($ mean $=3.9)$, P-value 0.001, which means that $\mathrm{Cr}$ level was significantly elevated among before-dialysis compared to after-dialysis. Also, there were significant differences in urea between before-dialysis (mean $=150.8$ ) and after-dialysis $($ mean $=67.1)$, P-value 0.001 , which means that urea was significantly elevated among before-dialysis compared to after-dialysis. In addition, there were significant differences in AST between before-dialysis (mean $=20.1$ ) and afterdialysis (mean $=24.8$ ), P-value 0.001, which means that AST was significantly lower among before-dialysis compared to after-dialysis. The results also show that there were significant differences in the ALT level between before- dialysis $($ mean $=20.7)$ and after-dialysis $($ mean $=25.1)$, Pvalue 0.001 , which means that ALT level was significantly lower among before-dialysis compared to after-dialysis. Furthermore, there were significant differences in the ALP level between before-dialysis (mean $=450.3$ ) and afterdialysis $($ mean $=484.4)$, P-value 0.003 , which means that ALP level was significantly lower among before-dialysis compared to after-dialysis. Also, there were significant differences in Ca level between before-dialysis (mean $=8.9$ ) and after-dialysis (mean $=9.2$ ), P-value 0.005, which means that $\mathrm{Ca}$ level was significantly lower among before-dialysis compared to after-dialysis. In addition, there were significant differences in phosphorus level between before-dialysis $($ mean $=4.9)$ and after-dialysis $($ mean $=4.0)$, P-value 0.001, 
which means that phosphorus was significantly elevated among before-dialysis compared to after-dialysis.

Table 7. Comparison of some biochemical parameters between before-dialysis and after-dialysis.

\begin{tabular}{llllll}
\hline \multirow{2}{*}{ Variables } & before-dialysis $(\mathbf{n}=\mathbf{4 3})$ & post-dialysis $(\mathbf{n}=\mathbf{4 3})$ & \multirow{2}{*}{ Mean differences } & \multirow{2}{*}{ t value } & \multirow{2}{*}{$\boldsymbol{P}$-value } \\
\cline { 2 - 4 } & Mean \pm SD & Mean \pm SD & -4.6 & -20.901 & 0.001 \\
Creatinine $(\mathrm{mg} / \mathrm{dL})$ & $8.5 \pm 1.9$ & $3.9 \pm 1.0$ & -83.7 & -23.230 & 0.001 \\
Urea $(\mathrm{mg} / \mathrm{dL})$ & $150.8 \pm 35.8$ & $67.1 \pm 24.5$ & 4.7 & 6.192 & 0.001 \\
AST (U/L) & $20.1 \pm 9.0$ & $24.8 \pm 10.3$ & 4.4 & 6.574 & 0.001 \\
ALT (U/L) & $20.7 \pm 16.3$ & $25.1 \pm 18.6$ & 34.1 & 3.172 & 0.003 \\
ALP (U/L) & $450.3 \pm 437.0$ & $484.4 \pm 459.2$ & 0.3 & 15.262 & 0.001 \\
Ca (mg/dL) & $8.9 \pm 0.3$ & $9.2 \pm 0.3$ & -0.8 & -10.836 & 0.001 \\
P (mg/dL) & $4.9 \pm 0.7$ & $4.0 \pm 0.4$ & & & \\
\hline
\end{tabular}

\section{Discussion}

One of the progressive CRF causing irreversible fall in the GFR further resulting in the increasing in kidney function levels [12]. Hypertension, T2DM, autoimmune diseases causes form the common cause of CRF [13]

Most patients undergoing hemodialysis were between the ages of 53 to 74 years, which could be due to advancing age and progressive physiological glomerulosclerosis that leads to a lowering in renal weight. Kidney function test were estimated in CKD patients before and after hemodialysis as these are important renal biomarkers in the diagnosis and good in follow-up of CKD patients undergoing Hemodialysis.

A rise in $\mathrm{Cr}$ levels in CRF patients is attributed to the decrease in the number of functioning nephrons, The rise in serum $\mathrm{Cr}$ is used in clinical marker of reduced GFR. HD decreases the $\mathrm{Cr}$ levels by its removal from the blood by dialyzing the patient's serum by containing minerals similar to the natural concentration of blood [13-14].

Our study showed that the before-dialysis group showed that increase and there were significant differences in urea and $\mathrm{Cr}$ level $(\mathrm{P}<0.001)$ when compared to healthy individuals. Also, the after-dialysis group showed significant elevated urea and $\mathrm{Cr}$ levels in after-dialysis groups when compared with healthy subjects. Urea and $\mathrm{Cr}$ levels in the after-dialysis group are lower than the after-dialysis group and are statistically significant ( $\mathrm{p}$-value $<0.05$ ). Our results agree with the previous study which found that the beforedialysis group showed an increase in the levels of the urea and $\mathrm{Cr}$ which were statistically significant ( $\mathrm{p}$-value $<0.05)$. Our results of $\mathrm{Cr}$ in the study group were consistent with the clinical studies done by other studied [8, 15-17]. These results confirm that HD is an efficient method to remove undesired substances from CKD patients, even when values are above the desired reference levels. This is due to the fall in the GFR in CRF patients. As the GFR falls, plasma levels of $\mathrm{Cr}$ and urea rise as they are eliminated by glomerular filtration and tubular secretion. Similarly, $\mathrm{Cr}$ and urea levels in the before-dialysis group were significantly higher in comparison with the after-dialysis group ( $\mathrm{p}$-value $<0.05$ ) [13].

In CRF, there is a steady and continued decrease in renal clearance or GFR, which leads to the gathering of urea and other toxic chemicals in the blood. The increase of serum urea is proportional to the progression of the disease, but it is highly influenced by the catabolic state or excessive protein ingestion [18]. Our findings of urea were supported by other studies and they found the decrease in the level of urea, $\mathrm{Cr}$ in after-dialysis patients compared with before-dialysis patients with CRF is due to the HD process which removes toxins from the blood by a closed-loop process where the blood of the patient and is continuously being withdrawn, dialyzed, and returned to the patient. These findings also supported by other studies $[8,19-$ 21].

The mean and standard deviation (SD) of serum calcium levels before-HD is $8.9 \pm 0.3$ and after-HD is $9.2 \pm 0.3$. The values are highly significant with $p$-value $=0.001$. Calcium levels in the blood of both males and females of CKD of after HD patients are increased slightly than the before-HD patients. Our findings are similar to previous study serum which showed that serum calcium levels in the after-dialysis group were higher in the before-dialysis group (p-value < 0.05 ). Serum calcium levels in the before-dialysis group were lower [13]. Bech et al., found a decrease in serum calcium levels after-dialysis [10]. Also, the previous study showed that serum phosphorus levels in the before-dialysis group were statistically significantly higher in comparison with the after-dialysis group ( $p$-value $<0.05$ ) [13].

Our study showed that the before-dialysis group showed that decrease and there were significant differences in ALT and AST levels $(\mathrm{P}<0.001)$ when compared to healthy individuals. Also, the after-dialysis group showed decreased serum levels of ALT and AST when compared with control and were statistically significant. Serum ALT and AST levels in the after-dialysis group are lower than the before-dialysis group and are statistically significant ( $\mathrm{p}$-value $<0.05$ ). This result agreed with the previous study which found that levels of liver enzyme, ALT activity between cases and healthy subjects participated in this study showed that decreasing levels of Alanine transaminase in patients with renal failure under HD when compared with control. As well as AST which showed a decrease in levels of Aspartate transaminase in patients with renal failure under HD when compared with control. Also, the levels of (AST\&ALT) concentration were significantly decreased in before-dialysis when compared to after dialysis for ALT \& AST [22]. Our study is also consistent 
with the previous study which showed that serum levels of the enzyme's ALT \& AST in patients with renal failure on hemodialysis; were decreased [23]. This result also agreed with previous study which found that both AST\&ALT were significantly low (P-value $<0.05$ ) in both sexes when compared with their counterparts in the control group the decrease in levels of aminotransferases in patients with renal failure who have not undergone hemodialysis therapy has observed in this study suggests that renal impairment has an impact on the liver enzymes [24]. In our results showed that a significant increase in the mean of the level of serum ALP in patients with renal failure groups compared to the control group. As well as a significant increase in the mean of the level of serum ALP in patients after HD compared to the control group. Also, the level of ALP concentration was significantly decreased in before-dialysis when compared to after-dialysis. Our study agreed with the previous study which found that ALP was significantly higher in both groups of CKD patients when compared to healthy controls [25].

In the previous study, subjects were considered as anemic on the basis of WHO criteria of $\mathrm{Hb}$ less than $13 \mathrm{~g} / 100 \mathrm{ml}$ for males and less than $12 \mathrm{~g} / 100 \mathrm{ml}$ for females [26]. The present study was conducted on $43 \mathrm{CKD}$ patients and 43 healthy controls and found that significantly mean RBCs count, hemoglobin concentration and hematocrit levels were significantly lower in both groups of CKD patients than normal healthy controls $(\mathrm{P}<0.05)$. This study agrees with the previous study which showed that significantly lower RBC count, hemoglobin concentration, and hematocrit levels in CKD patients when compared to healthy subjects [25]. Another recent work was consistent with our results and found that hemoglobin concentration and RBCs count were significantly different from that of the control at the severe CKD patients in Nigeria [27]. Our work indicated that the mean of each RBCs count, Hb, Hct, and RDW levels show a statistically significant increase in renal failure patients' afterHD when compared to before-HD levels. Our reports are consistent with the previous study which revealed that shows differences between the mean of RBCs count, Hb, Hct, RDW, RBCs indices in renal failure patients before and after HD [29]. The previous study indicated that the mean of each RBCs count, $\mathrm{Hb}, \mathrm{Hct}, \mathrm{MCH}, \mathrm{MCHC}$ and RDW levels show a statistically significant increase in renal failure patients' afterHD when compared to before-HD levels. The increase of each RBCs count, Hb, Hct levels after-HD were explained by the fact that before HD, patients are usually hypervolemic and the values of each RBCs count, $\mathrm{Hb}$, Hct levels are also lower [29].

The results of our study noticed that there was a significant change of WBC count in before-dialyzed and after-dialyzed patients compared to control but after-hemodialysis was different when compared to before-dialysis but not reach to significant. This finding is the disagreement that of other studies [25]. A study of Alghythan \& Alsaeed, 2012 revealed an insignificant difference in WBC count between hemodialyzed CKD patients and the control group [25]. The possible mechanism by which chronic renal disease leads to a slight decrease in total leukocytic count may be explained by complement activation in vivo due to exposure of blood to artificial membranes in dialyzer in patients undergoing dialysis. The complement is typically $\mathrm{C} 3 \mathrm{a}$ or $\mathrm{C} 5 \mathrm{a}$, produced by the classical complement activation pathway. Complement activation stimulates neutrophil aggregation and adherence to the endothelial surface which results in decreased leukocytic count. In patients undergoing hemodialysis, the incidence of this effect may be as high as $20 \%$ [30].

In the present work showed that platelets counts were significantly higher in before-dialyzed CKD patients compared to hemodialyzed $\mathrm{CKD}$ patients $(\mathrm{P}<0.05)$ and significant difference in platelet count was seen between before-dialyzed or hemodialyzed CKD patients and normal healthy controls ( $p=0.001 ; p=0.001$, respectively). Inversely previous study showed that there was a non-significant difference in PLT count of before-dialyzed CKD patients or hemodialyzed-CKD patients when compared with control group $(\mathrm{P}=0.052 ; \mathrm{P}=0.09$, respectively $)$ but our work consistent with this study that platelets counts were significantly higher in before-dialyzed CKD patients compared to hemodialyzed CKD patients $(P<0.05)$ [28]. Also Inversely the previous study revealed that platelets count in before-dialysis and after- hemodialysis no change $(p=0.319)$ [31].

A previous study compatible with our study showed that there was a significant difference in PLT count of beforedialyzed CKD patients when compared with control group $(p=0.0001)$ [32]. Similar results with our study also reported that there was a statistically significant decrease in the mean platelet counts, though still within the normal range, failure patients before- and after-HD when compared to the results of the control group. The mean platelet counts showed a significant decrease in patients' after-HD when compared to before-HD procedures [29].

The MCH level showed a statistically significant decrease in before-HD patients when compared to that of the control group $(\mathrm{P}<0.05)$. However, the MCV and RDW levels did not show any statistically significant change in before-HD patients compared to the control group ( $p>0.05$ ). our findings close for the previous study which showed that The $\mathrm{MCH}$ and MCHC levels showed a statistically significant decrease in before-HD patients when compared to that of the control group $(\mathrm{P}<0.05)$. However, the $\mathrm{MCV}$ and RDW levels did not show any statistically significant change in beforeHD patients compared to the control group ( $p>0.05$ ) [29].

\section{Conclusion}

From the present study, it can be concluded that patients with chronic kidney disease show abnormal hematological parameters, precisely reduced levels of RBC count, hemoglobin, hematocrit, and platelet count, in addition, before-dialysis patients are more anemic and thrombocytopenic than after-dialysis patients. These findings expose CKD patients to a higher risk of anemia-related complications and bleeding disorders, which may have a role 
in increasing the rate of patient mortality and morbidity. Moreover, hypertension and diabetes mellitus is the most common cause of CKD, and this is may indicate of lack of blood pressure and T2DM control in these patients. Liver enzymes activities vary in concentration pre and bost hemodialysis and make the after-dialysis patients are higher than before-dialysis.

\section{References}

[1] Yang, F., Zhang, L., Wu, H., Zou, H., \& Du, Y. (2014). Clinical analysis of cause, treatment and prognosis in acute kidney injury patients. PLoS One, 9 (2), e85214.

[2] Santoro, D., Caccamo, D., Lucisano, S., Buemi, M., Sebekova, K., Teta, D., \& De Nicola, L. (2015). Interplay of vitamin $\mathrm{D}$, erythropoiesis, and the renin-angiotensin system. BioMed research international, 2015.

[3] Alexander, R. T., Hemmelgarn, B. R., Wiebe, N., Bello, A., Morgan, C., Samuel, S., \& Tonelli, M. (2012). Kidney stones and kidney function loss: a cohort study. Bmj, 345, e5287.

[4] Bindroo, S., \& Challa, H. J. Review from StatPearls Publishing, Treasure Island (FL), 08 Aug 2018.

[5] de Francisco, A. L. M. (2010). Future directions in therapy for chronic kidney disease. Nefrología (English Edition), 30 (1), $1-9$.

[6] Vadakedath, S., \& Kandi, V. (2017). Dialysis: a review of the mechanisms underlying complications in the management of CRF. Cureus, 9 (8).

[7] Islam, M. N., Ferdous, A., Zahid, A. Z., Alam, M., \& Islam, M. N. (2015). Haematological profile of patients with chronic kidney disease in Northern Bangladesh. Dinajpur Med Col J, 8 (1), 21-7.

[8] Amin, N., Mahmood, R. T., Asad, M. J., Zafar, M., \& Raja, A. M. (2014). Evaluating urea and Cr levels in CRF before and after dialysis: a prospective study. Journal of cardiovascular disease, 2 (2), 1-4.

[9] Maduell, F., Rodriguez, N., Rodríguez, N., Arias Guillén, M., Arias-Guillén, M., Jimenez, S.,... \& Fontseré, N. (2012). Dialysate calcium individualisation: a pending issue. Nefrología (English Edition), 32 (5), 579-586.

[10] Bech, A., Reichert, L., Telting, D., \& de Boer, H. (2013) Assessment of calcium balance in patients on hemodialysis, based on ionized calcium and parathyroid hormone responses. J Nephrol, 26 (5), 925-930.

[11] Kendrick, J. and M. Chonchol (2011). "The role of phosphorus in the development and progression of vascular calcification." American Journal of Kidney Diseases 58 (5): 826-834.

[12] Tomás, I., Marinho, J. S., Limeres, J., Santos, M. J., Araújo, L., \& Diz, P. (2008). Changes in salivary composition in patients with renal failure. archives of oral biology, 53 (6), 528-532.

[13] Meenakshi, G. (2016). "Effect of hemodialysis on certain biochemical parameters in CRF patients." International Journal of Contemporary Medical Research 3 (10): 28692871.
[14] Ahmad, S., Misra, M., Hoenich, N., \& Daugirdas, J. (2008). HD Apparatus en Handbook of Dialysis.

[15] Shankalia, D. and A. Tanna (2013). "Role of HD in renal failure to correct biochemical parameters." Indian J Appl Res 3 (6): 414-416.

[16] Khaled M. D., A. F., A. T. Abdelgader, S. Bioprabhu. (2015). "effect of haemodialysis on some metabolic products of ckd patients in Libya." 4 (12): 45-54.

[17] Chielle, E. O., Rigon, K. A., Arcari, I. A., Stein, V., \& Santos, G. A. D. (2015). Influence of HD on the plasma concentration of adenosine deaminase in patients with chronic kidney disease. Jornal Brasileiro de Patologia e Medicina Laboratorial, 51 (3), 153-157.

[18] Montini, G., Pisanello, L., Testa, S., Daccò, V., Strologo, L. D., Taioli, E.,... \& Ardissino, G. (2003). Urea percentiles in children with CRF. Pediatric Nephrology, 18 (3), 261-265.

[19] Mohammed-Jumaah, I. A. (2013). "A study of some biochemical parameters in blood serum of patients with CRF." Journal of Basrah Researches (Sciences) 39 (4A): 20-32.

[20] Mahomoodally, M. F., \& Nugessur, H. (2014). before-and after-Dialysis Correla ons of Serum $\alpha$-Amylase, Crea nine and Urea in CRF Pa ents. Journal of Medical Research and Development (JMRD), 151-60.

[21] Mustafa, G., Khan, I. U. K. M. K., \& Sualeh, M. (2010). Antioxidant level in normal and dialyzed patients using FRAP method. Pakistan journal of pharmaceutical sciences, 23 (2).

[22] Babekr, A. A. S. (2015). The Effect of HD on Alanine Transaminase And Aspartate transaminase (ALT\&AST) levels in Patients with Renal Failure, Sudan University of Science and Technology.

[23] Sette, L. H. B. C. and E. P. de Almeida Lopes (2014). "Liver enzymes serum levels in patients with chronic kidney disease on hemodialysis: a comprehensive review." Clinics 69 (4): 271-278.

[24] Shittu, M. O., Adelakun, A., Eegunjobi, A., Idowu, O., \& Shittu, B. T. (2014). Analysis of aminotransferases in predialysis chronic kidney disease patients. IOSR J. Dent. Med. Sci, 13, 87-89.

[25] Latiwesh, O. B., Younis, M. Y., Shakila, S., Abdulmalik, F., Alammari, J. A., Min, Y.,... \& Peela, J. R. (2018). Hepatic enzymes changes in chronic kidney disease patients-a need for modified reference values. journal of evolution of medical and dental sciences-jemds, 7 (16), 1949-1954.

[26] Abate, A., Birhan, W., \& Alemu, A. (2013). Association of anemia and renal function test among diabetes mellitus patients attending Fenote Selam Hospital, West Gojam, Northwest Ethiopia: a cross sectional study. BMC Blood Disorders, 13 (1), 6.

[27] Afshar, R., Sanavi, S., Salimi, J., \& Ahmadzadeh, M. (2010). Hematological profile of chronic kidney disease (CKD) patients in Iran, in pre-dialysis stages and after initiation of hemodialysis. Saudi Journal of Kidney Diseases and Transplantation, 21 (2), 368.

[28] Latiweshob, O. B., Elwerfaly, H. H., \& Sheriff, D. S. (2017). Haematological changes in predialyzed and hemodialyzed chronic kidney disease patients in libya. IOSR J of Dental and Med Sciences, 16, 106-12. 
[29] Alghythan, A. K. and A. H. Alsaeed (2012). "Hematological changes before and after hemodialysis." Scientific Research and Essays 7 (4): 490-497.

[30] Walts., R. (2004). "Neutropenia. Wintrobe's Clinical Hematology 11th edition." Philadelphia, Lippincott Williams \& Wilkins 2 (63): 1748.

[31] Bat, T., Bat, B. E., El - Moghraby, A., Patel, S., Feng, X.,
Dunbar, C. E., \& Sarac, E. (2016). Thrombopoietic status of patients on haemodialysis. British journal of haematology, 172 (6), 954-957.

[32] Dorgalaleh, A., Mahmudi, M., Tabibian, S., Khatib, Z. K., Tamaddon, G. H., Moghaddam, E. S.,... \& Moradi, E. (2013). Anemia and thrombocytopenia in acute and CRF. International journal of hematology-oncology and stem cell research, 7 (4), 34. 